\title{
Multi-phase field simulation of multi-grain peritectic transition in multiple phase transformation
}

\author{
*Li Feng ${ }^{1,2}$, Jun-he Zhong1, Chang-sheng Zhu², Jun Wang ${ }^{1}$, Guo-sheng An', Rong-zhen Xiao ${ }^{1,2}$ \\ 1. College of Materials and Engineering, Lanzhou University of Technology, Lanzhou 730050, China \\ 2. State Key Laboratory of Advanced Processing and Recycling of Non-ferrous Metals, Lanzhou University of Technology, \\ Lanzhou 730050, China
}

\begin{abstract}
Taking Fe-C binary alloy as an example, based on the multi-phase field model, the nucleation and growth of $\delta$ phase, peritectic reaction, peritectic transformation, and the growth of subsequent austenite are simulated. Effects of the nucleation site of austenite on the peritectic reaction rate and the starting time of the peritectic transformation were studied. The simulation results show that the $\mathrm{Y}$ phase, as a shell, surrounds the $\delta$ phase and grows rapidly when the peritectic reaction occurs between the dendritic $\delta$ grains, and a layer of $Y$ phase shell is formed around $\delta$ phase after the peritectic reaction. After the $\delta$ phase is surrounded by $Y$ phase completely, the membrane shell separates the $L$ phase from the $\delta$ phase, so that the phase transfers from peritectic reaction to peritectic transformation. During the peritectic transformation, since the solute diffusion coefficient of the liquid phase is much greater than that of the solid phase, the average growth rate of austenite in the liquid phase is visibly higher than that of the $\delta$ phase. The peritectic reaction rate is related to the curvature of the nucleation site of the $y$ phase on the $\delta$ phase grains. The peritectic reaction rate at the large curvatures is faster than that at small curvatures.
\end{abstract}

Key words: phase field; Fe-C binary alloy; peritectic transformation; microstructure; numerical simulation CLC numbers: TP391.9 Document code: A

Article ID: 1672-6421(2020)05-357-07

$\mathrm{M}$ icrostructure determines the mechanical properties and service life of the castings, which makes it of great significance to effectively control the formation of microstructure in the forming process of the casting. This is a complex process, involving high temperature heat transfer, convection diffusion, solute diffusion, and the interaction between metal and mold, which is difficult to control and observe directly ${ }^{[1,2]}$. With the rapid development of computer software and hardware technology, the computer numerical simulation of the solidification process has become an important method for the research of solidification microstructure ${ }^{[3]}$. The phase field method is one of the common numerical simulation methods. The advantage of the phase field method is that there is no need to track complex solid/ liquid interface, and it is easy to couple with other external fields. The physical parameters of materials can be effectively expressed in the model in the form of phenomenological parameters, and the evolution of the solid/liquid interface and dendrite growth morphology

\section{*Li Feng}

Male, born in 1981, Associate Professor. His research interests mainly focus on the numerical simulation of microstructure in the solidification process.

E-mail: fenglils@lut.edu.cn;

Received: 2019-11-29; Accepted: 2020-05-03 can be accurately simulated ${ }^{[4,5]}$.

The peritectic phase transition is a common transition process in alloy phase transformation, and it is also one of the research hotspots of alloy solidification microstructure. In Kerr's study ${ }^{[6]}$, the peritectic phase transition process is described as two independent processes: peritectic reaction and peritectic transformation. The peritectic reaction is the reaction between the liquid phase and solid phase to form a new solid phase, and when the new solid phase separates the liquid phase from the original solid phase, the peritectic reaction ends. Peritectic transformation is the growth process that the austenite, the product of the peritectic reaction, enters the liquid phase and the primary solid phase after the end of the peritectic reaction. Phelan's research ${ }^{[7]}$ showed that the peritectic reaction is the process in which the $\mathrm{L}$ phase reacts with $\delta$ phase to produce austenite, and the reaction formula is $L+\delta \rightarrow \gamma$. The reaction is controlled by the solute diffusion in the $\mathrm{L}$ phase. The reaction speed is very fast, so that the austenite film can rapidly separate the $\delta$ phase from the $\mathrm{L}$ phase. Pan et al. ${ }^{[8]}$ simulated the growth of the austenite tip of a $\mathrm{Fe}-\mathrm{C}$ alloy peritectic reaction product based on the phase field method, and the effect of supercooling degree on the growth rate and morphology of the trijunction was studied. Li et al. ${ }^{[9]}$ established a phase 
field model of peritectic phase transition, which was used to simulate the growth behaviour of the nuclei of a single peritectic phase of a Ti-Al alloy attached to the surface of primary phase. The influence of super-cooling degree and initial composition on the extension of trijunction was studied. Based on the phase field method, Su et al. ${ }^{[10]}$ took a Ti-Al alloy as an example, and simulated the evolution of peritectic microstructure under the condition of continuous nucleation of small diameter samples and multiple nucleation of large diameter samples by using the peritectic phase transition model. Janin ${ }^{[1]}$ simulated the microstructure evolution in the process of peritectic transition of a Fe-C alloy based on the multi-phase field method, and studied the influence of the primary shape of $\delta$ phase on the morphology of the peritectic reaction product.

To date, the simulation research work on continuous phase transition process has been rarely reported. In this work, taking $\mathrm{Fe}-\mathrm{C}$ alloy as an example, the continuous micromorphology evolution in the processes of $\delta$ phase nucleation and growth $(\mathrm{L} \rightarrow \delta)$, peritectic reaction $(\mathrm{L}+\delta \rightarrow \gamma)$, and peritectic transformation $(\delta \rightarrow \gamma$, and $\mathrm{L} \rightarrow \gamma)$ was simulated. The effect of the curvature of austenite nucleation site on $\delta$ grain on the peritectic reaction rate and the starting time of the peritectic transformation was also discussed.

\section{Phase field model}

\subsection{Phase field control equation}

The governing equation of the phase field is as follows ${ }^{[12]}$ :

$$
\frac{\partial \phi}{\partial}=\sum_{\alpha \neq \beta}^{m} K_{\alpha \beta} \cdot \frac{2}{n} \sum_{j \neq i}^{n} S_{i j} M_{i j}\left(\frac{\delta F}{\delta \phi_{i}}-\frac{\delta F}{\delta \phi_{j}}\right)
$$

where, $\phi_{i}$ and $\phi_{j}$ are the phase field parameters of phases $i$ and $j$, representing different phase structures, $K$ is the time sequence function, $M_{i j}$ is the interface migration rate, $S_{i j}$ is the space step function ${ }^{[13]}, m$ is the number of phase transition undergone in the process, $F$ is the system free energy, the subscripts $\alpha$ and $\beta$ respectively represent liquid-solid phase transition and peritectic phase transition in multiple phase transition, and the subscripts $i$ and $j$ respectively represent the new phase and parent phase in the liquid-solid phase transition and peritectic phase transition.

The free energy $F$ of the system is specifically defined as follows:

$$
F=\int_{\Omega} f^{C H}+f^{T}+f^{E L}+f^{G B}
$$

where, $f^{G B}, f^{C H}$, and $f^{E L}$ denote the interfacial, component, and elastic free energy densities, respectively; $f^{T}$ represents the free energy density relating to a specific temperature, $\Omega$ is an integral of the density functional over the domain ${ }^{[14]}$. The expressions are as follows:

$$
\begin{gathered}
f^{C H}=\sum_{i=1}^{N} \phi_{i} f^{i}\left(c_{i}\right) \\
f^{T}=h(\phi) G_{i}^{r e g}+\left((1-h(\phi)) G_{j}^{r e g}+W g(\phi)\right.
\end{gathered}
$$

$$
\begin{gathered}
f^{E L}=\frac{1}{2}\left(\varepsilon^{i j}-\varepsilon^{* i j}\right) C^{i j k l}\left(\varepsilon^{k l}-\varepsilon^{* k l}\right) \\
f^{G B}=\sum_{i, j=1}^{N} \frac{4 \sigma_{i j}}{\eta_{i j}}\left\{\frac{\eta_{i j}^{2}}{\pi^{2}}\left|\nabla \phi_{i} \cdot \nabla \phi_{j}\right|+W_{i j}\right\}
\end{gathered}
$$

where, $f^{\mathrm{i}}\left(c_{i}\right), C^{i j k l}, \varepsilon, \eta_{i j}, \sigma_{i j}$, and $W_{i j}$ represent the component free energies of solute $c$ in phase $i$, the effective elasticity matrix, the phase-field gradient coefficient, interfacial thickness, interfacial energy, and potential well-function, respectively, $W$ is the excess free energy, and $g(\phi)$ is the imposed excess free energy function, which is given by $g(\phi)=$ $\phi^{2}\left(\phi^{2}-2 \phi-1\right)$. In addition, $G^{\text {reg }}$ denotes the Gibbs free energy, $G^{r e g}=\sum_{i=1}^{2}\left(c_{i} \mu_{i}^{0}+R T c_{i} \ln c_{i}\right)+\sum^{2} c_{i} c_{j} \sum^{n}\left(c_{i}-c_{j}\right), W_{i j}=\phi_{i} \phi_{j}$ $\left(0<\phi_{i}<1,0<\phi_{j}<1\right)$. To simplify the model, the influence of the stress and strain fields is neglected in the composition of $F$. Therefore, the free energy $F$ was changed to:

$$
F=\int_{\Omega} f^{G B}+f^{C H}+f^{T}
$$

\subsection{Solute control equation}

According to the law of solute conservation, the solute governing equation is obtained as ${ }^{[15]}$ :

$$
\frac{\partial c}{\partial t}=\nabla \cdot D_{i} \sum_{i} \phi_{i} \nabla C_{i}
$$

where, $D_{i}$ is the solute diffusion coefficient, and $C_{i}(i=1,2,3)$ respectively represent the solute concentrations of $\mathrm{L}$ phase, $\delta$ phase and $\gamma$ phase during solidification process.

\section{Phase field parameters and model numerical calculation}

\subsection{Phase field parameters}

During dendrite solidification process, the phase field equation ${ }^{[12]}$ is:

$$
\begin{aligned}
\frac{\partial \phi}{\partial t} & =M_{\delta}\left(\varepsilon(\theta)^{2} \nabla^{2} \phi+\frac{\partial}{\partial x}\left(|\nabla \phi|^{2} \varepsilon(\theta) \frac{\partial \varepsilon(\theta)}{\partial\left(\phi_{x}\right)}\right)\right. \\
& \left.+\frac{\partial}{\partial y}\left(|\nabla \phi|^{2} \varepsilon(\theta) \frac{\partial \varepsilon(\theta)}{\partial\left(\phi_{y}\right)}\right)-f_{\phi}\right)
\end{aligned}
$$

The expression for the migration rate $M_{\delta}$ of the dendrite solidification process is:

$$
\begin{gathered}
M_{\delta}^{-1}=\frac{\varepsilon^{3}}{\sigma \sqrt{2 W}}\left(\frac{1}{D} \zeta\left(c_{i}^{e}, c_{j}^{e}\right)\right) \\
\varepsilon=\sqrt{\frac{6 \lambda \sigma_{i j}}{2.2}}, \quad W=\frac{6.6 \sigma_{i j}}{\lambda}
\end{gathered}
$$




$$
f_{\phi}=\frac{R T}{V_{m}} h^{\prime}(\phi) \ln \frac{\left(1-c_{j}^{e}\right)\left(1-c_{i}\right)}{\left(1-c_{i}^{e}\right)\left(1-c_{j}\right)}+W g^{\prime}(\phi)
$$

where, $\varepsilon, \lambda, D, c_{i, j}{ }^{e}, h^{\prime}(\phi), g^{\prime}(\phi)$ and $\sigma$ refer to gradient energy coefficient, interface thickness, diffusion coefficient, the solute concentration distribution of $i$ phase and $j$ phase in equilibrium, the first order partial differential of potential function $h(\phi)$, the first order partial differential of excess free energy $g(\phi)$, and interface energy, respectively. Where, $c_{i}^{e}=\frac{\left(T_{m}-T\right)}{m_{1}}, c_{j}^{e}=\frac{\left(T_{m}-T\right)}{m_{2}}$ ( $m_{1}, m_{2}$ are liquidus slope of the $\delta$ and $\gamma$ phase), $h(\phi)=\phi^{3}(10-$ $\left.15 \phi+6 \phi^{2}\right), \lambda=2.5 \Delta x, \zeta=0.5 \lambda$.

$$
\varepsilon(\theta)=\varepsilon_{0}(1+v \cos (k \theta)), \tan \theta=\phi_{y} / \phi_{x}
$$

where, $k$ is the anisotropy constant, usually $k=4, v$ is anisotropic strength coefficient, $\theta$ is the angle between the interface and the growth direction, and $T$ is the peritectic reaction temperature.

During the peritectic phase transformation process, the phase field equation ${ }^{[8]}$ is:

$$
\frac{\partial \phi_{i}}{\partial t}=-\frac{2}{N} \sum_{j \neq i} S_{i j} M_{i j}\left(\frac{\delta F}{\delta \phi_{i}}-\frac{\delta F}{\delta \phi_{j}}\right)
$$

where, the phase field order parameters $\phi_{i}(i=1,2,3)$ respectively denote the volume fraction of $\mathrm{L}, \delta$ and $\gamma$ phase during solidification, $\sum_{i} \phi_{i}=1(i=1,2,3)$. In the present model, the step function $S_{i j}=S_{i} S_{j}, N=\sum_{1}^{3} S_{i}$.
The expression for the migration rate $M_{i j}$ of the peritectic phase transformation process is:

$$
M_{i j}=\frac{V_{m}}{R T_{m}} \frac{4 \varpi_{i j} D_{j}}{15 a_{2} \varepsilon^{2}} \frac{1}{\left(1-k_{i j}\right)} \frac{1}{\left(C_{j, e}^{i}-C_{i, e}^{j}\right)}
$$

where, $a_{2}$ is the normal number for the free energy density function ${ }^{[16]}, a_{2}=0.6276, T_{m}$ is the temperature of peritectic phase transition, $k_{i j}$ is solute partition coefficient, $V_{m}$ is the molar volume, $R$ is the ideal gas constant, and the gradient energy coefficient $\varepsilon$ and the height of potential energy $\varpi$ are calculated by the boundary energy $\sigma_{i j}$ and the interface thickness $\lambda$. The expressions are as follows: $\varpi_{i j}=3 \sqrt{2}\left(\sigma_{i j} / \lambda\right)$, $\varepsilon=\sqrt{3 \sqrt{2} \sigma_{i j} \lambda}$

\subsection{Physical parameters of Fe-C binary alloy}

The physical parameters of Fe-C binary alloy are shown in Table 1.

\subsection{Initial and boundary conditions}

The simulation domain of the peritectic phase transition is set as a mesh of $1,200 \times 1,200$, and the boundary of the calculated region is set as the adiabatic boundary condition, i.e., a zero-Neumann boundary condition. The finite difference scheme is used to solve the control equation in the phase field model, and the time step $\Delta t$ and the space step $\Delta x$ (space step $\left.\Delta x=\Delta y=10^{-8} \mathrm{~m}\right)$ need to meet the following stability condition:

$$
\Delta t<\min \left\{\Delta x^{2} /\left(4 D_{0}\right), \Delta y^{2} /\left(4 D_{0}\right)\right\}
$$

\begin{tabular}{|c|c|}
\hline Parameters & Values \\
\hline Initial solute concentration, $c_{0}(\mathrm{~mol} . \%)$ & 0.79 \\
\hline Molar volume, $V_{\mathrm{m}}\left(\mathrm{m}^{3} \cdot \mathrm{mol}^{-1}\right)$ & $7.7 \times 10^{-6}$ \\
\hline Gas constant, $R\left(\mathrm{~J} \cdot \mathrm{mol}^{-1} \cdot \mathrm{K}^{-1}\right)$ & 8.314 \\
\hline $\mathrm{L}$ phase diffusion coefficient, $D_{\mathrm{L}}\left(\mathrm{m}^{2} \cdot \mathrm{s}^{-1}\right)$ & $5.2 \times 10^{-7} \exp \left(-5.0 \times 10^{4} / R T\right)$ \\
\hline$\delta$ phase diffusion coefficient, $D_{\delta}\left(\mathrm{m}^{2} \cdot \mathrm{s}^{-1}\right)$ & $1.27 \times 10^{-6} \exp \left(-8.3 \times 10^{4} / R T\right)$ \\
\hline Y phase diffusion coefficient, $D_{Y}\left(\mathrm{~m}^{2} \cdot \mathrm{s}^{-1}\right)$ & $7.61 \times 10^{-6} \exp \left(-13.7 \times 10^{4} / \mathrm{RT}\right)$ \\
\hline $\mathrm{Y} / \mathrm{L}$ interface energy, $\sigma_{12}\left(\mathrm{~J} \cdot \mathrm{m}^{-2}\right)$ & 0.319 \\
\hline$\delta / L$ interface energy, $\sigma_{23}\left(\mathrm{~J} \cdot \mathrm{m}^{-2}\right)$ & 0.204 \\
\hline $\mathrm{y} / \delta$ interface energy, $\sigma_{13}\left(\mathrm{~J} \cdot \mathrm{m}^{-2}\right)$ & 0.370 \\
\hline Peritectic temperature, $T_{\mathrm{m}}(\mathrm{K})$ & 1,768 \\
\hline Liquidus slope of the $\delta$ phase $m_{1}\left(\mathrm{~K} \cdot \mathrm{mol}^{-1}\right)$ & $-1,828$ \\
\hline Liquidus slope of the $y$ phase $m_{2}\left(\mathrm{~K} \cdot \mathrm{mol}^{-1}\right)$ & $-1,399$ \\
\hline Partition coefficient of the $\delta / L$ interface, $k_{\delta\llcorner}$ & 0.179 \\
\hline Partition coefficient of the $\mathrm{y} / \mathrm{L}$ interface, $k_{\mathrm{yL}}$ & 0.334 \\
\hline Partition coefficient of the $\mathrm{\gamma} / \bar{\delta}$ interface, $k_{\mathrm{y} \delta}$ & 1.865 \\
\hline
\end{tabular}

In the simulation of this work, the $\delta$ phase nucleated in the form of heterogeneous nucleation, and five $\delta$ phase nuclei

Table 1: Physical parameters of Fe-C binary alloy ${ }^{[8,17]}$ 
randomly distributed in the liquid phase, as shown in Fig. 1(a). Setting the initial nucleation radius of $\delta$ phase nucleus to be $\mathrm{R}$, after setting the initial temperature, the growth of $\delta$ grain is simulated and calculated. Figure 1(b) shows the growth morphology of $\delta$ phase at $t=15,000 \Delta t$. With the decrease of temperature, peritectic reaction occurs on the surface of dendritic $\delta$ phase, the $\gamma$ phase nucleates in the form of heterogeneous nucleation, and its initial nucleation radius was set as R1. When the peritectic reaction occurs, the initial conditions of $\mathrm{L}, \delta$ and $\gamma$ phases in the simulation region are shown in Figs. 1(c) and (d).
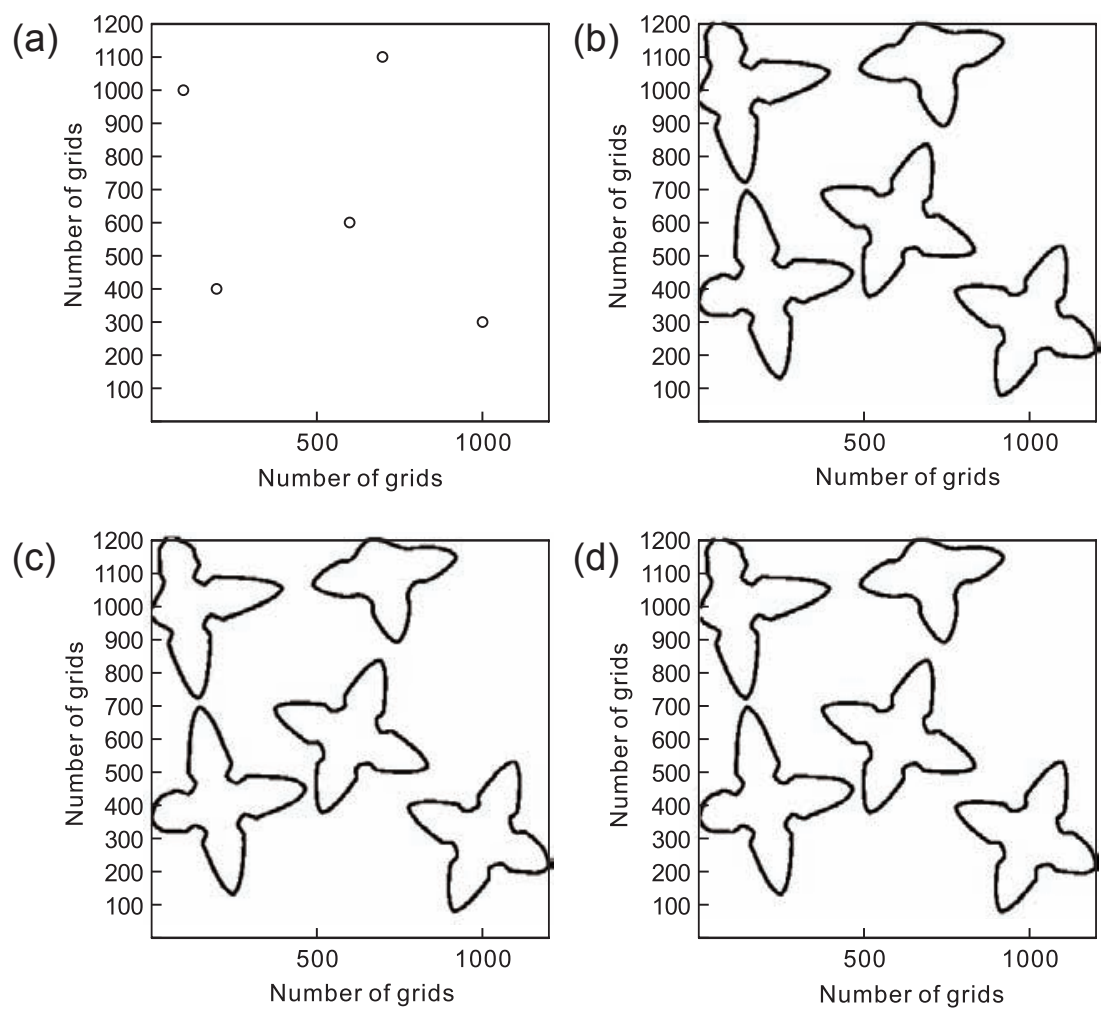

Fig. 1: Initial boundary conditions

\section{Simulation results and analysis}

\subsection{Peritectic reaction}

At $1,768 \mathrm{~K}$, a new solid $\gamma$ was formed in $\mathrm{Fe}-\mathrm{C}$ alloy by peritectic reaction between the liquid phase and $\delta$ phase, and the equation is $\mathrm{L}+\delta \rightarrow \gamma$. Before the peritectic reaction, the $\delta$ phase solidified in a dendritic shape from the liquid phase ${ }^{[18]}$, as shown in Fig. 1(b). When it reaches peritectic temperature $T_{\mathrm{P}}$, the peritectic reaction occurs on the surface of the $\delta$ phase. From the perspective of nucleation energy, there is a higher critical nucleation energy at the dendrite surface, which makes the austenite easier to nucleate. The heterogeneous nucleation of the $\gamma$ phase on the dendrite surface of the $\delta$ phase is easier than that of homogeneous nucleation in the liquid phase. Therefore, the $\gamma$ phase nucleates at the dendrite interface of the $\delta$ phase. The nucleation phenomenon in Figs. 1(c) and (d) is consistent with the Cahn's nucleation theory ${ }^{[19]}$. Figure 2 shows the simulation results of peritectic reactions on the surface of the dendrite $\delta$ phase at different times when the $\gamma$ phase is nucleated at the $\delta$ phase tip (i.e. at the interface of large curvature). Figure 2(ac) show the peritectic reaction results at $t=15,000 \Delta t, 25,000 \Delta t$, and $30,000 \Delta t$, respectively. It can be seen from the above figures that during the peritectic reaction, the $\delta$ phase is rapidly surrounded by $\gamma$ phase. The $\mathrm{L}$ phase is out of contact with $\delta$ phase when the original $\delta$ phase is surrounded completely by the new $\gamma$ phase. Figure 2(d-f) shows the carbon atom distributions of solute corresponding to Fig. 2(a-c), respectively. It can be seen from the distribution diagram and isogram of carbon atoms that the solute concentration at the interface of $\gamma / \delta$ increases gradually in the process of peritectic reaction. When the $\delta$ phase is surrounded completely by the $\gamma$ phase membrane shell, it is difficult for the solute atoms in the membrane shell to diffuse outward, resulting in an increase in the front concentration of the membrane shell growing towards the interior of $\delta$ phase. The $\gamma$ phase membrane shell separates the L phase from the $\delta$ phase, which makes the peritectic reaction difficult to continue, and the phase transfer changes from the peritectic reaction to a peritectic transformation.

\subsection{Peritectic transformation}

In $\mathrm{Fe}-\mathrm{C}$ alloy, a certain amount of liquid phase remains after the peritectic reaction, and this part of liquid phase crystallizes into austenite in the subsequent cooling process, which is mainly attributed to the less $\delta$ phase and more liquid phase before the peritectic reaction ${ }^{[20]}$. Figure 3 shows the morphology and solute distribution of the $\gamma$ phase grown into the parent phase 

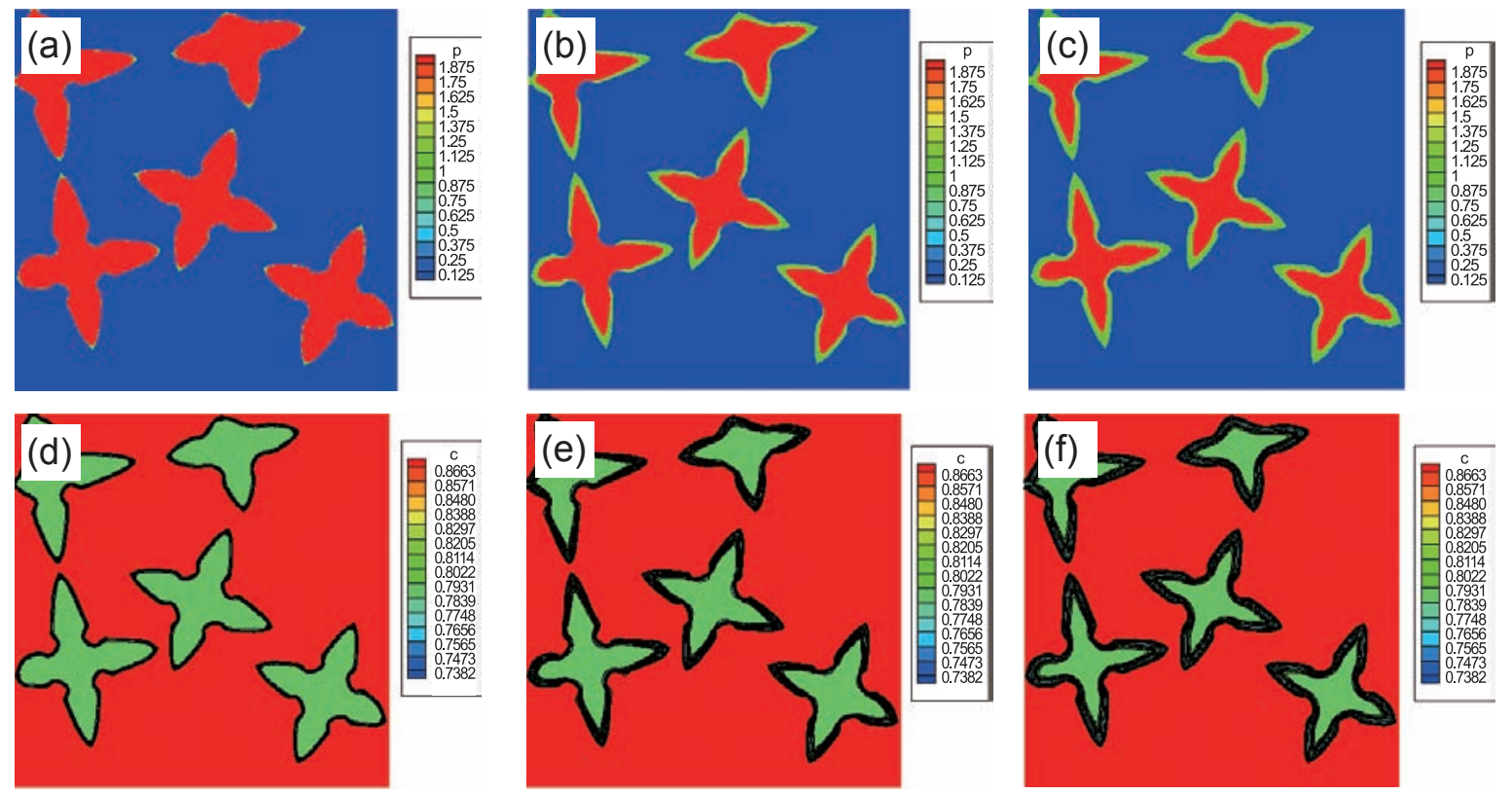

Fig. 2: Simulation results of peritectic reaction
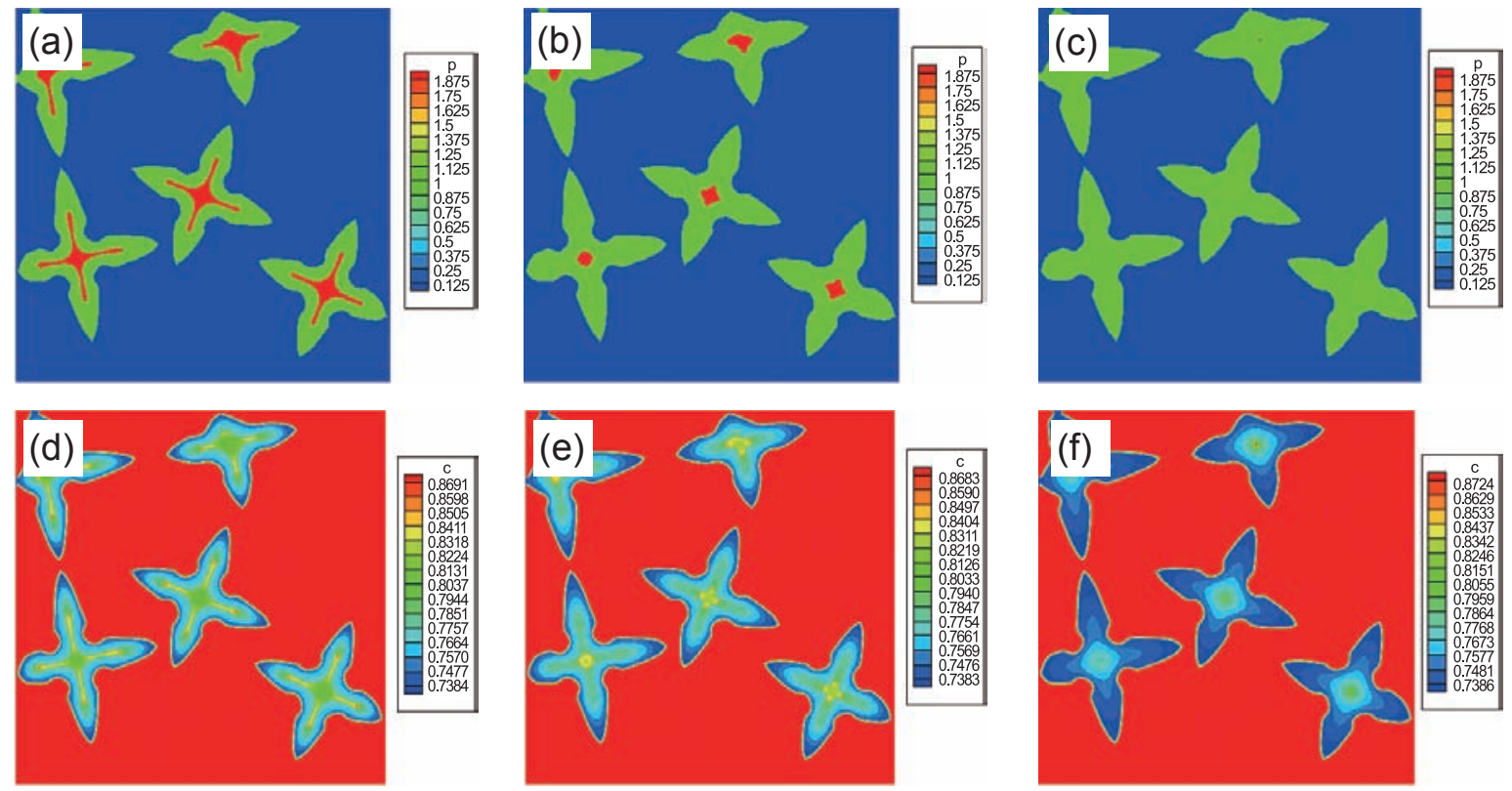

Fig. 3: Simulation results of peritectic transformation

at a time of $t=100,000 \Delta t, 160,000 \Delta t$, and 390,000 $\Delta t$. As can be seen from Fig. 3(a-c), with the development of peritectic transformation, the $\delta$ phase shrinks toward the center, and the thickness of membrane shell of $\gamma$ phase increases gradually. At the same time, the solute diffusion distance becomes greater and greater, and the transformation is becomes slower and slower. In the process of peritectic phase transition, the carbon atoms are easily diffused due to the higher temperature, and there is usually no primary $\delta$ phase in the final structure ${ }^{[21]}$. Figure $3(\mathrm{~d}-\mathrm{f})$ shows the distribution of solute at times corresponding to Fig. 3(a-c), respectively. It can be seen that the concentration in the core of the shell gradually increased, forming a solute-rich area due to the difficulty in outward diffusion of solute atoms in the $\gamma$ phase shell during the peritectic transformation, as shown in Figs. 3(d, e).
When the $\delta$ phase is completely transformed into $\gamma$ phase, the concentration at the core of the shell is large, and there is a large concentration gradient with the outside. Solute atoms will migrate from the high concentration area to the low concentration area, according to Fick's First Law. Thus, when the $\delta$ phase is completely transformed to $\gamma$ phase, the concentration of the core gradually decreases, as shown in Fig. 3(f).

Figure 4 shows the morphology and solute distribution of the $\gamma$ phase growing into L phase, and there is no $\delta$ phase in the solid phase. Figure 4(a) shows the morphology of $\gamma$ phase at $400,000 \Delta t$. It can be seen that the $\gamma$ dendrites grow continuously and some of them contact with each other. Figure 4(b) shows the morphology of $\gamma$ phase at $450,000 \Delta t$, and the $\gamma$ grains are in contact with each other. Where the dendrites are in contact, the 

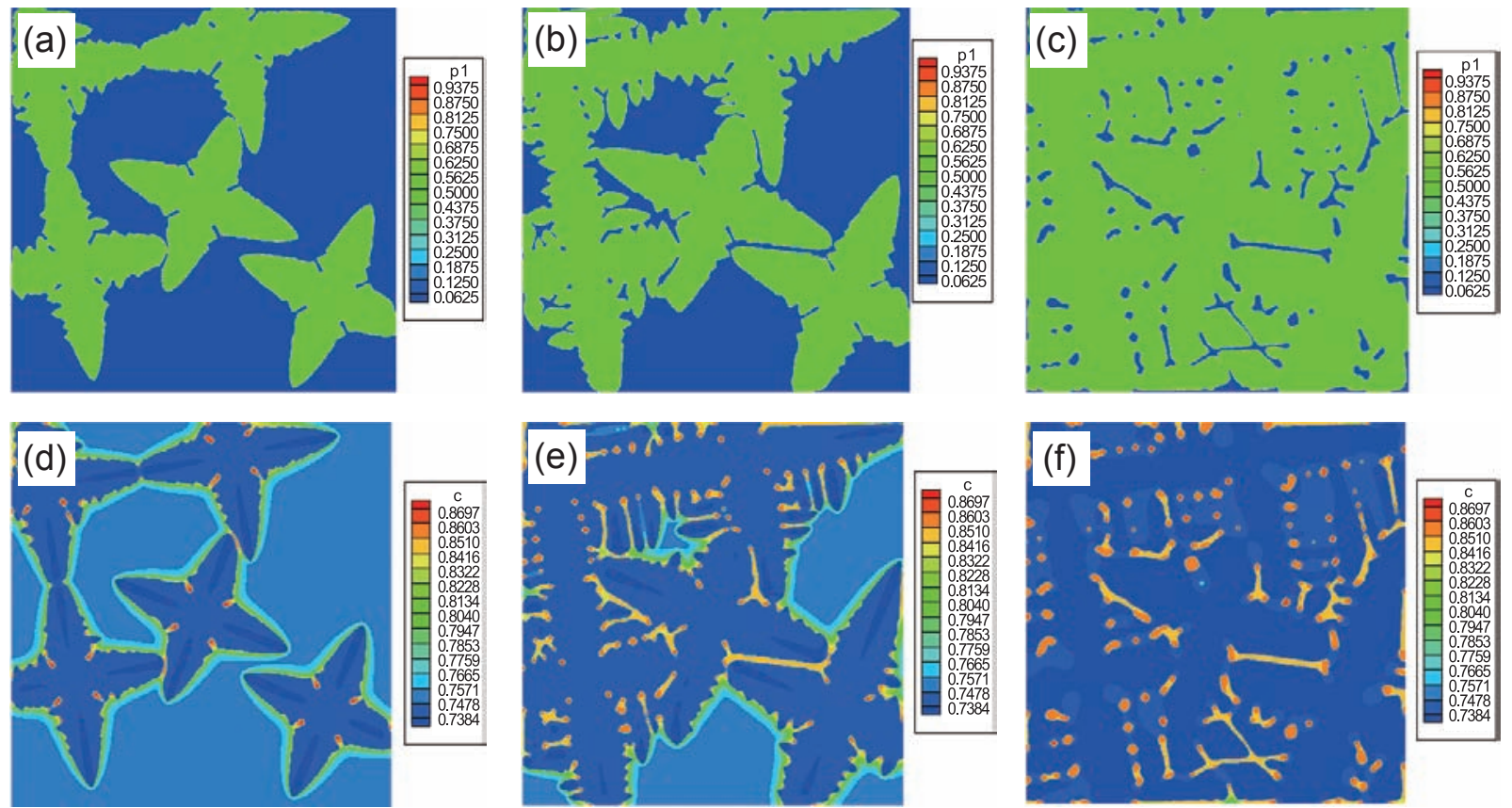

Fig. 4: Simulation results of y growth morphology

secondary and tertiary dendrite arms bend or stop growing due to the growth space being occupied. The growth of $\gamma$ grains is basically stopped at $470,000 \Delta t$, and the entire simulation area is filled with $\gamma$ grains. Figure $4(\mathrm{~d}-\mathrm{f})$ shows the carbon atom distribution at times corresponding to Fig. 4(a-c), respectively. It can be seen that the dendrite interface frontier edge is the brightest, indicating the solute concentration is the highest in this region. Due to the redistribution of solute atoms, the solute atoms precipitated during the solidification process cannot sufficiently diffuse into the liquid phase, so that the solute atoms are enriched at the dendrite interface frontier edge. In addition, the solute is found at a high concentration between the secondary and tertiary dendrite arms. Moreover, when the interface area is surrounded by dendrite and secondary dendrite arms, the carbon atom diffusion channel is blocked and cannot be diffused into the liquid phase, resulting in a relatively high concentration of carbon atoms in these areas, and eventually forming a solute trapping area, which reduces the melting point of these areas and the solidification rate is slower than that of other places.

Here, the rate multiplier $k$ was defined as the ratio of the average rate of the $\gamma$ phase growth into the $\mathrm{L}$ phase to that of the $\gamma$ phase growth into the $\delta$ phase in the peritectic transformation. The rate multiplier $k=5.35$ was obtained by calculation, which indicates that the average growth rate of $\gamma$ in the L phase is obviously faster than that in the $\delta$ phase. This is because the diffusion coefficient of solute atoms in the $\mathrm{L}$ phase is much greater than that in the $\delta$ phase $\left(D_{\mathrm{L}}>D_{\delta}\right)$.

\subsection{Effect of curvature of nucleation site on peritectic phase transformation}

In order to study the effect of curvature on the rate of peritectic reaction, two kinds of $\gamma$ phase nucleation sites were set. The first initial condition is shown in Fig. 1(c), where the $\gamma$ phase nucleation is set at the small curvature interface of $\delta$ phase. The second initial condition is shown in Fig. 1(d), where the $\gamma$ phase nucleation is set at the large curvature interface of $\delta$ phase. In the later peritectic transformation process, a heterogeneous nucleation method was adopted for the numerical simulation of an austenite nucleus.

Figure 5 shows the relationship between the peritectic reaction rate and time at different nucleation sites corresponding to Figs.1(c, d). It can be seen that at the beginning of the peritectic reaction, the curvature of the nucleation site has little effect on the peritectic reaction, and the values of the two curves in Fig. 5 are almost equal. This is because the $\gamma$ phase on the surface of the $\delta$ phase has the same contact area with the $\mathrm{L}$ phase, when the phase transition occurs, as the equation is $\mathrm{L}+\delta \rightarrow \gamma$, it consumes the same $\mathrm{L}$ phase. With the progress

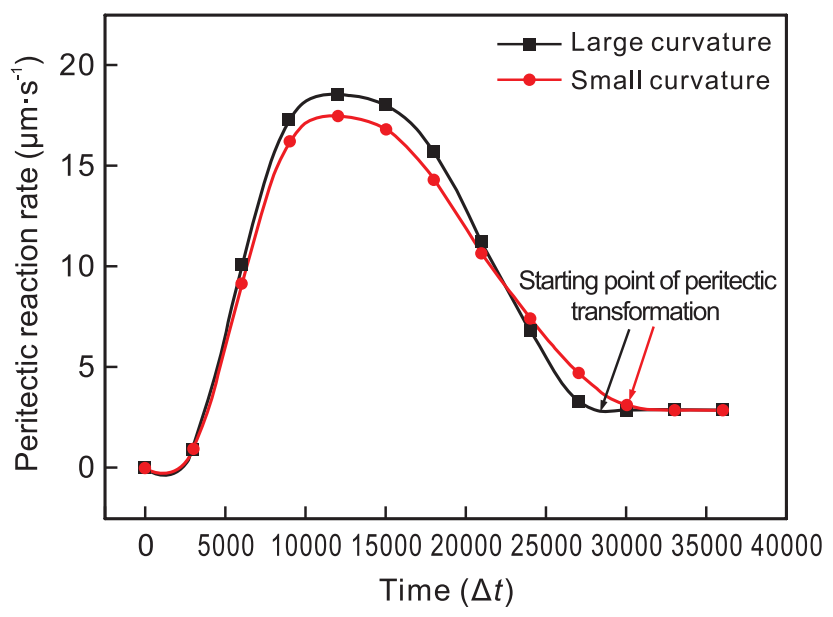

Fig. 5: Relationship between the rate and time of peritectic reaction 
of the peritectic reaction, the influence of curvature on the rate of peritectic reaction increases gradually, and the peritectic reaction rate under a large curvature is greater than that of a small curvature. At the time $t=30,000 \Delta t$, the rate of peritectic reaction under two kinds of curvatures is very low, the surface of $\delta$ phase is almost covered by $\gamma$ phase, and the peritectic reaction is almost complete. In both cases, the rate of peritectic reaction decreases to nearly zero. When nucleating at a site of a large curvature, the start time of the peritectic transformation is close to $28,000 \Delta t$. The start time of the peritectic transformation is close to $30,000 \Delta t$ when nucleating at a site of small curvature, the starting points of their peritectic transformations are shown by the black and red arrows in Fig. 5, respectively. This indicates that the peritectic transformation time under large curvatures is shorter than that under small curvatures. At the same time, it can be seen from the curve in Fig. 5 that the values of the reaction rates under the two different curvatures are equal after entering the peritectic transformation stage. This indicates that the curvature of the nucleation site only has a greater influence on the rate of peritectic reaction and has no obvious effect on the later peritectic transformation.

\section{Conclusions}

(1) When the peritectic reaction occurs on the surface of the dendritic $\delta$ phase, the $\gamma$ phase, as a shell, grows rapidly surrounding the $\delta$ phase. After the peritectic reaction is completed, a layer of $\gamma$ phase membrane shell is formed around the dendritic $\delta$ phase, and a layer of $\gamma$ phase shell is formed around $\delta$ phase after the peritectic reaction. After the $\delta$ phase is completely surrounded by $\gamma$ phase, the membrane shell separates the L phase from the $\delta$ phase so that the phase transits from peritectic reaction to peritectic transformation.

(2) The average growth rate of austenite in the liquid phase is significantly higher than that in $\delta$ phase during the peritectic transformation, which is related to the fact that the solute diffusion coefficient of the liquid phase is much greater than that of the solid phase.

(3) The rate of peritectic reaction is related to the curvature of the nucleation site of the austenite. The rate of peritectic reaction at a large curvature is faster than that at a small curvature, and the peritectic transformation occurs firstly at the large curvature nucleation site. The reaction rate values under the two different curvatures are equal after entering the peritectic transformation stage, indicating that the curvature of the nucleation site has a greater influence on the peritectic reaction rate, but has no obvious effect on the subsequent peritectic transformation.

\section{Acknowledgements}

This work was funded by the National Natural Science Foundation of China (Grant Nos.: 11504149, 51661020) and Natural Science Foundation of Gansu Province of China (Grant No.: 18JR3RA147).

\section{References}

[1] Feng Li, Lu Ni-ni, Gao Ya-long, et al. Phase-field simulation of secondary dendrites growth in directional solidification of binary alloys. China Foundry, 2019, 16(2): 97-104.

[2] Liu J K, Niu Y, Yan W, et al. Simulation of grain growth by phase field method. Journal of Xi'an University of Technology, 2008, 28(4): 360-365. (In Chinese)

[3] Tourret D, Karma A. Growth competition of columnar dendritic grains: A phase-field study. Acta Materialia, 2015, 82: 64-83.

[4] Feng L, Wang Z P, Lu Y, et al. Phase-field model of isothermal solidification of binary alloy with multiple grains. Acta Physica Sinica, 2008, 57(2): 1084-1090. (In Chinese)

[5] Zhang J, Chen W X, Zheng C W, et al. Phase field simulation of austenite-ferrite phase transition in Fe-C-Mn ternary alloy, Acta Physica Sinica, 2017(6): 122-130. (In Chinese)

[6] Kerr H W, Cisse J, Bolling G F. On equilibrium and nonequilibrium peritectic transformations. Acta Metallurgica, 1974, 22(6): 677-686.

[7] Phelan D, Reid M, Dippenaar R. Kinetics of the peritectic phase transformation: In-situ measurements and phase field modeling. Metallurgical \& Materials Transactions A, 2006, 37(3): 985-994.

[8] Pan S, Zhu M, Rettenmayr M. A phase-field study on the peritectic phase transition in Fe-C alloys. Acta Materialia, 2017,132: 565-575.

[9] Li X Z, Su Y Q, Guo J J, et al. Phase-field research of microstructure evolution for directionally solidified peritectic transition I. Extension of trijunction. Acta Metallurgica Sinica, 2006(6): 41-47. (In Chinese)

[10] Su Y Q, Li X Z, Guo J J, et al. Phase-field research of microstructure evolution for directionally solidified peritectic transition II. Simulation of nucleation-controlled microstructure. Acta Metallurgica Sinica, 2006, 42(6): 606-610. (In Chinese)

[11] Janin Tiaden. Phase field simulations of the peritectic solidification of Fe-C. Journal of Crystal Growth, 1999, 198: 1275-1280.

[12] Li F, Jia B B, Zhu C S, et al. Multi-phase field simulation of grain growth in multiple phase transformations of a binary alloy. Chinese Physics B, 2017(8): 91-99.

[13] Kim S G, Kim W T, Suzuki T, et al. Phase-field modeling of eutectic solidification. Journal of Crystal Growth, 2004, 261(1): 135-158.

[14] Steinbach I, Apel M. Multi-phase field model for solid state transformation with elastic strain. Physica D, 2006, 217(2): 153-160.

[15] Karma A. Phase-field formulation for quantitative modeling of alloy solidification. Physical Review Letters, 2001, 87(11): 115701.

[16] Karma A, Rappel W J. Quantitative phase-field modeling of dendritic growth in two and three dimensions. Physical Review E, 1998, 57(4): 4323-4349.

[17] Ohno M, Matsuura K. Quantitative phase-field modeling for two-phase solidification process involving diffusion in the solid. Acta Materialia, 2010, 58(17): 5749-5758.

[18] Hu H Q. Principle of metal solidification. Beijing: Machinery Industry Press, 2000: 200-203. (In Chinese)

[19] Cahn J W. The kinetics of grain boundary nucleated reactions. Acta Metallurgica, 1956, 4(5): 449-459.

[20] Cui Z Q, Tan Yanchun. Metallology and heat treatment principle. Beijing: Machinery Industry Press, 2007: 114-116 (In Chinese)

[21] Zu F Q, Xi Y, Li X Y. Peritectic solidification and its new understandings. Foundry, 2013, 62(2): 91-95. (In Chinese) 